Chirurg 2020 $91: 263$

https://doi.org/10.1007/s00104-020-01135-5

Online publiziert: 12. Februar 2020

(c) Springer Medizin Verlag GmbH, ein Teil von Springer Nature 2020

\section{Originalpublikation}

Hobeika C, Fuks D, Cauchy F et al (2020) Impact of cirrhosis in patients undergoing laparoscopic liver resection in a nationwide multicentre Survey. Br J Surg. https://doi.org/10. 1002/bjs.11406. [Epub ahead of print]

Einleitung. Leberteilresektionen bei hepatozellulärem Karzinom (HCC) in Leberzirrhose haben durch die Grunderkrankung des Leberparenchyms eine erhöhte postoperative Morbidität und Mortalität. Durch das laparoskopische, minimal-invasive Vorgehen soll durch Vermeidung des Absetzens von Kollateralen (Lig. umbilicale etc.) und des großen Zugangswegs die Inzidenz dieser Probleme deutlich gesenkt werden. In der hier vorgestellten retrospektiven multizentrischen Analyse über einen sehr langen Zeitraum der Association Française de Chirurgie wird dies landesweit im Vergleich zu solchen Eingriffen bei Patienten ohne Zirrhose aufgearbeitet.

Methodik. Es wurden alle erwachsenen Patienten ( $>18$ Jahren) der Jahre 2000 bis 2017, bei denen eine laparoskopische Leberteilresektion in 27 französischen Zentren durchgeführt wurde, in diese retrospektiven Analyse eingeschlossen. $\mathrm{Ab}$ einem histologischen Fibrosegrad 4 wurde eine Zirrhose definiert. Ein Cutoff des verbleibenden Restlebervolumens von $30 \%$ vs. $40 \%$ des Gesamtlebervolumens bei jeweils Nichtzirrhose und Zirrhose wurde angestrebt. Die 90-TageMorbidität und Mortalität wurden nach der Durchführung des „propensity score matching " zwischen die Patienten mit Zirrhose vs. ohne Zirrhose verglichen.

Aladdin Ali Deeb · Michael Ardelt ${ }^{1} \cdot$ Utz Settmacher

' Universitätsklinikum Jena, Klinik für Allgemein-, Viszeral- und Gefäßchirurgie, Jena, Deutschland

\title{
Leberzirrhose bei laparoskopischen Leberteilresektionen
}

Ergebnisse. Insgesamt wurden $3150 \mathrm{~Pa}$ tienten eingeschlossen. Davon hatten 774 Patienten (24,6\%) eine Leberzirrhose. Die 90-Tage-Mortalitätsrate lag bei Patienten mit Zirrhose bei 2,6\% vs. $0,6 \%$ ohne Zirrhose $(p=0,001)$. Die schwere Morbiditätsrate (Clavien-Dindo $\geq$ III) lag bei 10,6\% mit Zirrhose vs. $6,7 \%$ ohne Zirrhose $(p=0,001)$. In einer Multivariatanalyse war die Zirrhose ein unabhängiger Risikofaktor für die schwere Morbidität (Odds Ratio 2,12; $p=0,001$ ) sowie die Mortalität (Odds Ratio 4,61; $p=0,001)$. Im Rahmen des „propensity score matching“ wurden 282 Patienten mit Zirrhose gegenüber 282 Patienten ohne Zirrhose verglichen. Dabei zeigten die Patienten mit Zirrhose bei gleichen intraoperativen Parametern eine größere Tendenz zur Entwicklung schwerer Komplikationen (Odds Ratio $1,74 ; p=0,096)$ sowie ein höheres Mortalitätsrisiko (Odds Ratio 5,13; $p=0,036$ ).

Diskussion. Die landesweite Erfassung der Daten über diesen Zeitraum ist zunächst beachtlich. Obwohl das Resektionsausmaß in den „High-volume“und Expertenzentren höher war und auf fortgeschrittene Befunde schließen lässt, war die Mortalität dort bei gleicher Morbidität geringer. Die Aussagekraft dieser Analyse ist durch die Diskrepanz der unterschiedlichen Expertisen zwischen den verschiedenen Zentren und dem langen Untersuchungszeitraum sowie die retrospektive Studiennatur natürlich eingeschränkt. Es zeigt sich aber bei Analyse dieser Versorgungssituation in Frankreich im Unterschied zu Daten aus ausgewählten Hochleistungszentren, dass mit dem minimal-invasiven Vorge- hen der Risikofaktor der Leberzirrhose nicht $\mathrm{zu}$ vernachlässigen bleibt (insbesondere postoperatives Leberversagen und Mortalität), obwohl der Blutverlust, die Rate der schweren kardiopulmonalen Komplikationen, Galleleckagen, postoperative Blutungen und Revisionen bei den Patienten mit Leberzirrhose mit denen ohne Zirrhose vergleichbar waren.

\section{Kommentar}

Insgesamt eine sehr gute Zusammenstellung der Versorgungssituation der laparoskopischen Chirurgie primärer Lebertumoren in unserem Nachbarland mit der klaren Botschaft, dass auch das laparoskopische Vorgehen den Risikofaktor Zirrhose nicht eliminieren kann.

\section{Korrespondenzadresse}

PD Dr. med. habil. Dr. med. univ.

\section{Michael Ardelt}

Universitätsklinikum Jena, Klinik für Allgemein-, Viszeral- und Gefäßchirurgie

Am Klinikum 1, 07747 Jena, Deutschland

Michael.Ardelt@med.uni-jena.de

Interessenkonflikt. A. Ali Deeb, M. Ardelt und U. Settmachergeben an, dass kein Interessenkonflikt besteht. 